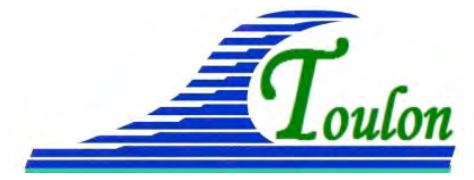

XIV èmes $\begin{gathered}\text { Journées Nationales Génie Côtier - Génie Civil } \\ \text { Toulon, } 29 \text { juin au } 1^{\text {er }} \text { juillet } 2016\end{gathered}$
$\begin{array}{ll}\text { DOI:10.5150/jngcgc.2016.036 } & \text { C Editions Paralia CFL } \\ \text { disponible en ligne - http://www.paralia.fr - available online }\end{array}$

\title{
Systèmes de mesure du transport sédimentaire en zone à fort courant
}

\author{
Olivier BLANPAIN ${ }^{1}$, Thierry GARLAN ${ }^{1}$, Jean François FILIPOT ${ }^{2}$, \\ France FLOC' ${ }^{3}$, Sandrine ROBIN ${ }^{4}$, Cyril GIRY ${ }^{5}$, \\ Yann MEAR ${ }^{6}$, Amandine NICOLLE ${ }^{7}$
}

1. Service Hydrographique et Océanographique de la Marine, équipe sédimentologie, 13, rue du Chatellier, CS92803, 29228 Brest Cedex 2, France. blanpain@shom.fr

2. France Energie Marine, 15 rue Johannes Kepler, 29200 Brest, France. jean.francois.filipot@france-energies-marines.org

3. IUEM, Domaines Océaniques UMR 6538, Place Nicolas Copernic, 29280 Plouzané, France.france.floch@univ-brest.fr

4. iXSurvey, 46, quai François Mitterrand, 13600 La Ciotat, France. sandrine.robin@ixsurvey.com

5. Energie de la Lune, 87 quai des Queyries, 33100 Bordeaux, France.

c.giry@energiedelalune.fr

6. Université de Caen, LUSAC, EA 4253 et Cnam/Intechmer, 60, rue Max Pol Fouchet, 50130 Cherbourg-Octeville, France. yann.mear@cnam.fr

7. ENSTA Bretagne, 2 Rue François Verny, 29200 Brest, France. amandine.nicolle@ensta-bretagne.fr

\section{Résumé :}

Dans le cadre de l'ANR PHYSIC (Processus Hydro-Sédimentaires en Interaction avec les Courants extrêmes), plusieurs systèmes instrumentaux vont être mis en œuvre dans le Raz Blanchard afin d'y qualifier et d'y quantifier la dynamique sédimentaire :

- la stéréoscopie vidéo : le couplage de deux caméras synchronisées, filmant la même scène permet d'obtenir les coordonnées dans les trois dimensions de chaque pixel de l’image et ainsi d'accéder à la taille et la vitesse de déplacement des particules;

- un mât de capteurs d'impacts : ce dispositif enregistre les impacts des particules à haute fréquence à plusieurs altitudes au-dessus du fond ;

- le suivi acoustique de particules marquées : des émetteurs acoustiques introduits dans des galets, dont les caractéristiques physiques sont parfaitement connues, permettent d'estimer leur position à une fréquence élevée ;

- un profileur acoustique multifréquences donnant accès à la concentration et à la taille des particules en suspension dans la colonne d'eau.

Mots-clés : Dynamique sédimentaire, Charriage, Suspension, Instrumentation, Raz Blanchard. 


\section{Introduction}

Les hydroliennes sont destinées à être déployées sur des sites à fort courant qui peuvent être sujets à un transport sédimentaire important. Ce flux de particules peut présenter une contrainte voire un verrou lors de l'exploitation d'un champ d'hydroliennes s'il n'est pas clairement caractérisé. Les rares études menées dans, ou plus exactement à proximité, de tels environnements hydro-sédimentaires extrêmes mettent en évidence une dynamique de particules grossières qui ne répond pas aux modèles de processus couramment admis. Il apparaît donc nécessaire d'acquérir de nouvelles données qui pourront s'avérer cruciales dans les phases de conception, d'installation, d'ancrage, de raccordement, d'exploitation et de maintenance des hydroliennes.

Dans ce but, une première phase de l'ANR PHYSIC, consiste à développer des systèmes instrumentaux spécifiques capables de répondre aux questions suivantes :

- Quels sont les modes de transport des différentes classes sédimentaires (sables, graviers, galets)?

- Quelle est l'amplitude et la variabilité spatiale et temporelle du débit solide charrié et en suspension?

- Quelle est la taille maximale des particules aux différentes altitudes au-dessus du fond?

Les réponses apportées doivent permettre aux concepteurs d'hydroliennes de prendre en compte les risques d'abrasion ou d'endommagement des structures immergées et d'affouillement ou d'accrétion autour de ces mêmes structures.

Plusieurs techniques instrumentales, identifiées comme prometteuses, sont présentées :

- la stéréoscopie vidéo ;

- un mât de capteurs d'impacts ;

- le suivi acoustique de particules marquées ;

- le profileur acoustique multifréquences.

Ces systèmes se doivent d'être opérationnels dans les conditions de mer les plus éprouvantes (fort coefficient de marée et forte houle) afin de pouvoir établir la capacité maximale d'agressivité du flux sédimentaire. Ils seront mis à l'eau dans le Raz Blanchard au début de l’année 2017.

\section{Caractéristiques hydro-sédimentaires du site de déploiement}

\subsection{Forçages physiques : vagues et courants de marée}

Au nord du Cotentin, les vagues peuvent atteindre 3 mètres voire plus lors du passage des dépressions hivernales. La direction de la houle la plus fréquente est le nord-ouest, avec une hauteur moyenne de 0,50 à 1,25 mètre. La synthèse des états de la mer, établie par Météo-France montre que 2\%o des états de mers correspondent à des Hs de 6 à 9 mètres. De plus, quand le courant porte au vent, on trouve dans le raz une mer très creuse et déferlante. 


\section{XIV èmes Journées Nationales Génie Côtier - Génie Civil \\ Toulon, 29 juin au $1^{\text {er }}$ juillet 2016}

Le Raz Blanchard est le secteur présentant les plus forts courants de marée des côtes françaises, et se trouve parmi les 11 secteurs les plus énergétiques du monde. Les courants de marée peuvent atteindre en surface des vitesses de 10 nœuds en vive-eau moyenne (modélisation TELEMAC 3D, SHOM 2013).

\subsection{Dynamique sédimentaire}

La couverture sédimentaire est caractérisée par un gradient de granularité allant de la roche dans la partie la plus énergétique à des bancs couverts de dunes de sables, en passant successivement par des fonds de cailloutis, de graviers et de sables grossiers (Figure 1).

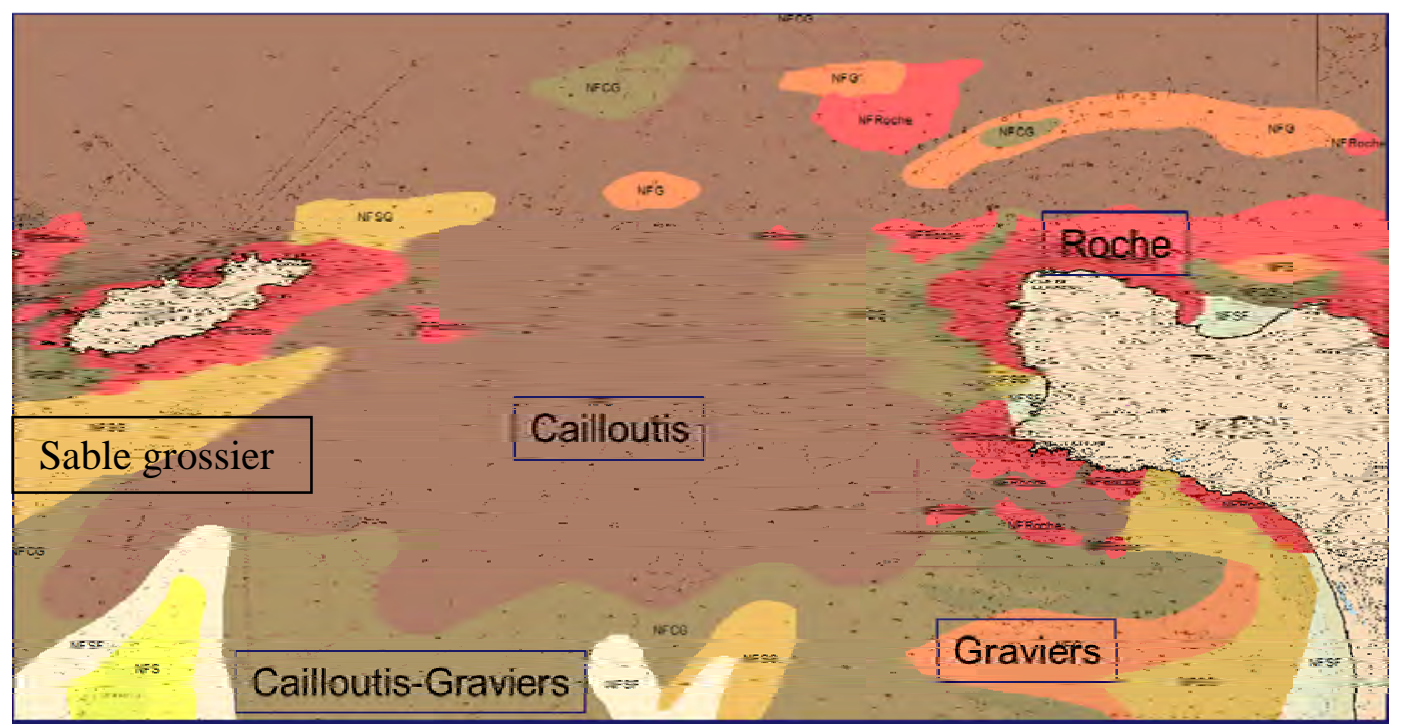

Figure 1. Carte sédimentaire des abords du Cap de la Hague (VASLET et al., 1978).

Les secteurs rocheux du Golfe normand-breton où les courants marins sont importants ont été qualifiés d'asédimentaires, c'est-à-dire que les sédiments ne peuvent s’y déposer et que la roche y affleure. Mais l'hydrodynamique extrêmement puissante et les stocks de galets d'origines marine et continentale indiquent plutôt une zone de transit de sédiments traversant le Raz Blanchard au gré des marées et des épisodes de tempêtes.

La granulométrie particulièrement grossière et étendue implique un arrangement du lit et un comportement hydraulique particuliers : phénomènes de pavage, d'entrave, de masquage et d'exposition. Or la connaissance du transport de telles couvertures sédimentaires soumises aux forts courants de marée et à la houle est extrêmement pauvre. En mer, les techniques instrumentales utilisées habituellement ne permettent pas d'étudier finement les mécanismes en jeu dans de telles conditions hydro-sédimentaires. Dans un environnement de moindre intensité, les mesures in situ acquises avec un instrument prototype, le DySPI (BLANPAIN et al., 2009), ont permis de sélectionner et calibrer des formulations traduisant les processus particulier de ces faciès (BLANPAIN 
2009). Les formules ont été intégrées dans un modèle de transport sédimentaire multiclasses et multicouches en Manche : un flux résiduel d'au moins un galet par mètre linéaire, transite dans le Raz Blanchard toutes les 10 minutes (Figure 2). Cependant, aucune donnée de terrain n'est venue valider ce résultat.

De plus, le forçage exceptionnellement intense qui a lieu dans le Raz Blanchard pourra induire d'autres phénomènes (saltation de galets, sheet-flow de sédiments grossiers...) qui, à notre connaissance, n’ont jamais été documentés.

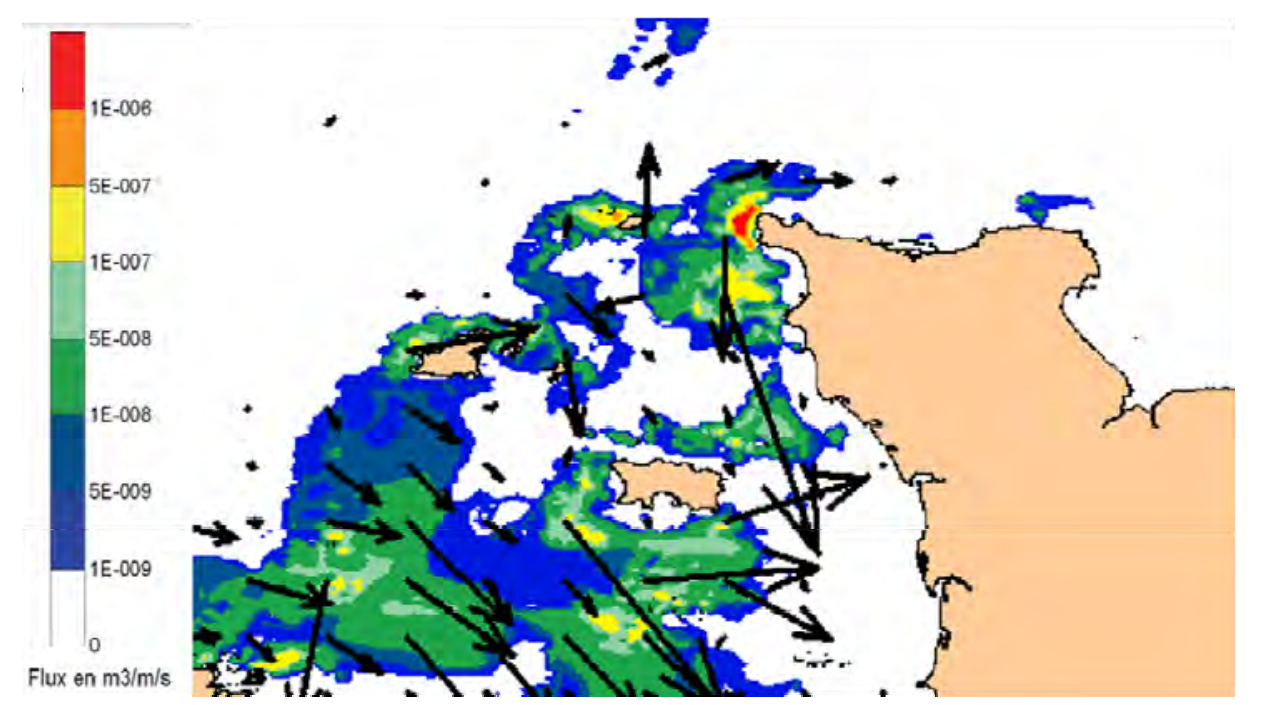

Figure 2. Modélisation des flux sédimentaires résiduels des galets en Manche (BLANPAIN, 2009).

\section{Descriptif des systèmes}

Pour pouvoir observer ces mécanismes et les traduire numériquement, l'acquisition des données doit se faire à haute résolution : à l'échelle spatiale de la particule, et à l'échelle temporelle de la turbulence, ce que ne permettent pas la plupart des techniques classiques d'étude du transport sédimentaire. Les systèmes développés seront ainsi équipés des dernières générations de capteurs optiques et acoustiques et seront détournés afin d'être capable de caractériser la dynamique hydro-sédimentaire à haute fréquence. Trois techniques innovantes sont développées ci-dessous. Conjointement, des capteurs plus classiques seront également déployés :

- Deux ADCPs de fréquence différentes. Ils seront placés verticalement pour fournir des profils de vitesse sur toute la colonne d'eau, déterminer les paramètres de vagues et fournir les propriétés turbulentes de l'écoulement.

- Un profileur acoustique multifréquences. Il donne accès à la concentration et à la taille des particules en suspension sur une certaine tranche de la colonne d'eau en inversant les signaux rétrodiffusés. 


\section{XIV èmes Journées Nationales Génie Côtier - Génie Civil \\ Toulon, 29 juin au $1^{\text {er }}$ juillet 2016}

Pour faciliter leur mise en œuvre et la sécurité lors des opérations à la mer, plusieurs structures relativement simples et de dimensions raisonnables seront privilégiées plutôt qu'une seule comportant tous les systèmes. Afin de ne pas émettre de bruit parasite et de favoriser la stabilité des structures au fond, aucun marquage de surface ne sera utilisé. La conception des structures se devra de minimiser les perturbations du courant (de flot et de jusant) pour obtenir un jeu de données représentatif des conditions locales et d'être robuste pour résister aux flux sédimentaires. On s'orientera donc vers des structures profilées hydrodynamiquement, renforcées aux extrêmes et suffisamment lestées.

Une campagne de tests sera réalisée à l'automne 2016 dans un secteur de moyenne énergie afin de :

- valider le déroulement des opérations à la mer,

- valider la conception de la structure porteuse (architecture, fiabilité du système, des capteurs...),

- valider la pertinence des données acquises (perturbations, résolution, redondance...),

- valider le protocole d'analyse et de la chaîne de traitement des données.

\subsection{Stéréoscopie vidéo}

Les caractéristiques intrinsèques du capteur définissent les résolutions spatiale et temporelle obtenues :

- A une distance de 1,3 m de la caméra, la surface filmée mesurera 1,92 m par 1,08 m et chaque pixel correspondra à un carré de $1 \mathrm{~mm}$ de côté. Seuls l'angle de vue et le nombre de pixels conditionnent la résolution spatiale.

- Une particule se déplaçant à $5 \mathrm{~m} / \mathrm{s}$ parcourra $8,3 \mathrm{~cm}$ entre deux images acquises à $60 \mathrm{~Hz}(20 \mathrm{~cm}$ à $25 \mathrm{~Hz})$. Seule la fréquence d'acquisition conditionne la résolution temporelle.

Cette dernière considération montre l'intérêt d'un temps de pose nettement inférieur au laps de temps entre deux images pour pourvoir distinguer le contour les particules en mouvement. Une vitesse d'obturation rapide est donc nécessaire, ce qui implique un éclairage important ou une grande sensibilité du capteur.

Le couplage de deux (ou plus) caméras synchronisées, filmant la même scène (stéréoscopie), permet d'obtenir les coordonnées dans les 3 dimensions de chaque point commun identifié sur chacune des images (ASTRUC et al., 2012 ; MUSUMECI et al., 2013). Cette technique nécessite au préalable une calibration minutieuse des caractéristiques intrinsèque de chaque caméra (distance focale, coefficient de distorsion...) ainsi qu'un mesure précise de la position et de l'orientation relative des deux caméras. Un post traitement des images est ensuite nécessaire pour rectifier les images, identifier les points communs et reconstruire une image dont les coordonnées de chaque pixel identifié sont connues. L'analyse de ces images permet par exemple de déterminer la taille de chaque particule en suspension identifiée, sa forme et sa hauteur 


\section{Thème 3 - Instrumentation, mesures, imagerie et télédétection}

au-dessus du fond quelle que soit sa distance à la caméra. Par corrélation temporelle réalisée sur plusieurs images successives, la vitesse de déplacement des particules peut ensuite être mesurée.

L'erreur obtenue sur la détermination de chacune des trois coordonnées lors de la triangulation sera diminuée pour chaque pixel en fonction du nombre de caméras qui permettent de l'identifier et de l'angle formé entre les caméras.

A notre connaissance, ce dispositif n’a jamais été utilisé pour étudier le transport sédimentaire en milieu marin.

\subsection{Mât de capteurs d'impacts}

Bien que les hydrophones soient couramment utilisés en milieu marin (bruit ambiant, mammifères marins), ils le sont très peu pour étudier le charriage. C'est néanmoins un instrument qui a fait ses preuves en rivières, dans des conditions de vitesses de courant et de couverture sédimentaire qui se rapprochent de celles rencontrées dans nos zones d'intérêts. Une étude réalisée en 2014 par le SHOM sur une plage du Morbihan et sur le banc du Four a montré le potentiel de cette technique d'acoustique passive pour l'étude de la dynamique des sables en milieu marin (BLANPAIN et al., 2015).

Les géophones sont utilisés en rivière depuis des dizaines d'années pour estimer le débit solide charrié (RICKENMANN et al., 2013). Le dispositif, installé sur le lit de la rivière, se compose typiquement de plusieurs plaques métalliques en dessous des quelles sont fixés des hydrophones. Les impacts sonores des particules y transitant sont enregistrés à haute fréquence. A notre connaissance, aucun dispositif de la sorte n’a été utilisé en mer. Notre intérêt ici n'est pas de disposer les plaques résonnantes sur le fond mais verticalement, afin d'obtenir une discrétisation de l'altitude au-dessus du fond. Ce système de mesure est à rapprocher des capteurs d'impacts couramment utilisés dans l'étude du transport éolien de sable. Une étude préalable pour déterminer l'influence de la taille, de la forme et de la vitesse d'impact des particules sur le signal produit est nécessaire.

\subsection{Suivi acoustique de particules marquées}

Le système de traçage décrit ici se distingue de ceux utilisés depuis des dizaines d'années (fluorescence, magnétisme, puces RFID...) car il propose un suivi quasi continu de la position des particules. Il s'appuie sur des appareils émetteurs et récepteurs couramment utilisés pour marquer des poissons (BERGE et al., 2012) ou localiser des équipements sous l'eau.

Un émetteur est intégré dans chaque particule choisie pour l'intérêt/la pertinence de ses caractéristiques (taille, forme, densité...). La mise en œuvre de plusieurs dizaines de particules marquées permettra de couvrir une large gamme de caractéristiques physiques différentes. Cette façon de faire a été utilisée avec succès en rivières avec des puces RFID passives (DICKSON et al., 2011; CHAPUIS, 2012). Une attention particulière 


\section{XIVìmes Journées Nationales Génie Côtier - Génie Civil \\ Toulon, 29 juin au $1^{\text {er }}$ juillet 2016}

doit être apportée à ne pas modifier les caractéristiques physiques des particules. Chaque émetteur à une signature propre, ce qui permet d'individualiser le traçage. Une fois les particules immergées, elles émettent un signal acoustique à un pas de temps défini.

Pour s’affranchir des risques de désynchronisation des horloges des récepteurs et des émetteurs, la position de ces derniers sera estimée en fonction de la différence entre les dates d'arrivée du signal aux différents récepteurs (principe inverse de la navigation hyperbolique ou méthode de multilatération). Le signal doit être capté par au moins trois antennes réceptrices du maillage réalisé au fond pour localiser l'émetteur précisément.

La précision sur le calcul des distances dépend à la fois de la précision sur la mesure du temps de parcours de l'onde et sur la vitesse du son dans l'eau. Cette dernière est fonction de la température, de la salinité et de la pression, grandeurs qui devront donc être mesurées (sonde CTD) sur zone.

La méthode proposée implique de connaître la position exacte de chaque antenne réceptrice posée au fond. Ceci est réalisable en utilisant un système de positionnement acoustique basé sur la triangulation (méthode dite par " temps de vol ») à partir d'un bateau en surface : les antennes réceptrices sont équipées d'un émetteur, le bateau d'un récepteur dont la position est connue précisément grâce au DGPS. La position relative de chaque particule dans le repère d'antennes réceptrices au fond peut ainsi être reliée à une position absolue dans un repère géodésique.

\section{Conclusions}

Les lacunes de connaissance de la dynamique sédimentaire dans les sites à fort courant proviennent de la difficulté à y effectuer des mesures. Les conditions hydrosédimentaires intenses rencontrées dans le Raz Blanchard se situent par exemple en dehors du domaine de validité des formulations de transport classiquement utilisées.

La volonté récente de développer des fermes hydroliennes dans ces zones engendre ainsi des questions qui restent sans réponse: les flux sédimentaire locaux sont-ils admissibles par les hydroliennes et les infrastructures associées (câbles, connecteurs...)? Afin d'y répondre, des systèmes de mesure spécifiques, capables de mesurer les paramètres critiques dans des environnements marins très énergétiques vont être développés et déployés. Ces systèmes seront équipés des dernières générations de capteurs optiques et acoustiques pour caractériser la dynamique hydro-sédimentaire à haute fréquence. L'expérience montre que le cumul de plusieurs systèmes au sein d'un même programme de mesures est efficace pour atteindre le but recherché. Ainsi, des ADCPs, un système de caméra stéréoscopique, des capteurs d'impacts, un système de suivi acoustique de particules marquées et un profileur acoustique multifréquences seront mis en œuvre. 
Thème 3 - Instrumentation, mesures, imagerie et télédétection

\section{Références bibliographiques}

ASTRUC D., CAZIN S., CID E., EIFF O., LACAZE L., ROBIN P., TOUBLANC F., CACERES I. (2012). A strereoscopic method for rapid monitoring of the spatiotemporal evolution of the sand-bed elevation in the swash zone. Coastal Engineering, Vol. 60, pp. 11-20. http://dx.doi.org/10.1016/j.coastaleng.2011.08.007

BERGE J., CAPRA H., PELLA H., STEIG T., OVIDIO M., BULTEL E., LAMOUROUX N. (2012). Probability of detection and positioning error of a hydro acoustic telemetry system in a fast-flowing river: Intrinsic and environmental determinants. Fisheries Research, Vol. 125-126, pp 1-13. http://dx.doi.org/10.1016/j.fishres.2012.02.008

BLANPAIN O. (2009). Dynamique sédimentaire multiclasse: de l'étude des processus à la modélisation en Manche. Thèse Université de Rouen.

BLANPAIN O., BAILLY DU BOIS P., CUGIER P., LAFITE R., LUNVEN M., DUPONT J., LEGRAND J., LE GALL E., PICHAVANT P. (2009). Dynamic sediment profile imagery (DySPI): a new field device for the study of dynamic processes at the sediment-water interface. Limnology and Oceanography: Methods, Vol. 7, pp 8-20. http://dx.doi.org/10.4319/lom.2009.7.8

BLANPAIN O., DEMOULIN X., WAELES B., RAVILLY M., GARLAN T., GUYOMARD P. (2015). Passive acoustic measurement of bedload discharge features on a sandy seafloor. Seabed and Sediment Acoustics: Measurements and Modelling. Proceedings of the Institute of Acoustics. Vol. 37. Pt.1. Bath, 7-9 septembre 2015.

CHAPUIS M. (2012). Mobilité des sédiments fluviaux grossiers dans les systèmes fortement anthropisés : éléments pour la gestion de la basse vallée de la Durance. Thèse de l'Université d'Aix - Marseille.

DICKSON M., KENCH P., KANTOR M. (2011). Longshore transport of cobbles on a mixed sand and gravel beach, southern Hawke Bay, New Zealand. Marine Geology, Vol. 287, pp 31-42. http://dx.doi.org/10.1016/j.margeo.2011.06.009

MUSUMECI R., FARINELLA G., FOTI E, BATTIATO S, PETERSEN T., SUMER M. (2013). Measuring sandy bottom dynamics by exploiting depth from stereo video sequences. A. Petrosino (Ed.): ICIAP 2013, Part I, LNCS 8156, pp 420-430. http://dx.doi.org/10.1007/978-3-642-41181-6_43

RICKENMANN D., TUROWSKI J., FRITSCHI B., WYSS C., LARONNE J., BARZILAI R., REID I., KREISLER A., AIGNER J., SEITZ H., HABERSACK H. (2013). Bedload transport measurements with impact plate geophones: comparison of sensor calibration in different gravel-bed streams. Earth Surf. Process. Landforms. http://dx.doi.org/10.1002/esp.3499

VASLET D., LARSONNEUR C., AUFFRET J.P. (1978). Carte des sédiments superficiels de la Manche. BRGM, France, 1/500 000. 